\title{
Effects of probiotics on experimental necrotizing enterocolitis: a systematic review and meta-analysis
}

\author{
Gayatri Athalye-Jape ${ }^{1,2,3}$, Shripada Rao ${ }^{1,3}$ and Sanjay Patole ${ }^{2,3}$
}

BACKGROUND: Meta-analyses of randomized controlled trials (RCTs) suggest that probiotics decrease the risk of necrotizing enterocolitis (NEC) in preterm infants. Many animal RCTs have evaluated probiotics for preventing NEC. We systematically reviewed the literature on this topic.

METHODS: The protocol for systematic review of animal intervention studies (SYRCLE) was followed. Medline, Embase, ISI Web of Science, e-abstracts from the Pediatric Academic Society meetings, and other neonatal conferences were searched in December 2015 and August 2016. RCTs comparing probiotics vs. placebo/no probiotic were included.

RESULTS: A total of 29 RCTs were included (Rats: 16, Mice: 7, Piglets: 3, Quail: 2, Rabbit: 1; $N \sim 2,310$ ), with 21 reporting on histopathologically confirmed NEC; remaining 8 assessed only pathways of probiotic benefits. Twenty of the 21 RCTs showed that probiotics significantly reduced NEC. Pooling of data was possible for 16/21 RCTs. Meta-analysis using random-effects model showed that probiotics significantly decreased the risk of NEC (203/641 (31.7\%) vs. 344/571 (60.2\%); relative risk: 0.51; 95\% confidence interval (Cl): 0.42-0.62; $P<0.00001 ; 12=44 \%$; number needed to treat: 4; $95 \% \mathrm{Cl}: 2.9,4.3)$.

CONCLUSION: Probiotics significantly reduced NEC via beneficial effects on immunity, inflammation, tissue injury, gut barrier, and intestinal dysbiosis.

\section{INTRODUCTION}

$\mathbf{N}$ ecrotizing enterocolitis (NEC) is a devastating gastrointestinal emergency in mostly preterm infants (gestation $<32$ weeks) with significant mortality $(25 \%)$ and morbidity, including long-term neuro-developmental impairment (1-11). Mortality (45-100\%) and morbidity is highest in infants born before 28 weeks of gestation (12-14). The economic burden associated with $\geqslant$ Stage II NEC has been estimated to be as high as one billion dollars per year in United States of America, not accounting for the expenses associated with ongoing care of survivors of NEC with neurodevelopmental impairment $(15,16)$. Despite extensive research over decades, there is currently no cure for the condition because the pathogenesis of NEC is not clearly understood
$(17,18)$. Prevention of NEC is hence a priority given the substantial health burden associated with the condition.

Meta-analyses of randomized controlled trials (RCTs) have suggested that probiotic supplementation significantly decreases the risk of $\geqslant$ Stage II NEC in human preterm infants (19-22). The proposed mechanisms for the beneficial effects of probiotics include enhancement of gut barrier, immune response modulation (e.g., Toll-like receptor 4 receptor, nuclear factor-B, inflammatory cytokines), and competitive inhibition of gut colonization by pathogens to limit dysbiosis (23-27).

Studies in animal models are crucial for understanding the mechanisms for the benefits and adverse effects of an intervention selected for potential clinical use (28-30). Investigators have evaluated the effects of probiotics in different animal models of NEC, but the sample sizes of individual animal studies are usually small. Meta-analysis of data from small but comparable individual studies is a valuable method to generate reliable evidence with higher precision and power $(31,32)$. Systematic reviews of animal studies can enable the translation of findings to clinical trials rapidly and appropriately while ensuring effective use of time $(33,34)$. Hence, we decided to systematically review studies in animal models to evaluate the efficacy of probiotic supplementation in decreasing the risk of NEC and to understand the pathways for benefits of probiotics in reducing the incidence and/or severity of the illness.

\section{Aim \\ To conduct a systematic review of studies assessing the effects of probiotics in animal models of NEC. \\ METHODS \\ The SYRCLE protocol for systematic review of animal intervention studies was followed (Systematic Review Protocol for Animal Intervention Studies Format by SYRCLE (WWW.SYRCLE.NL). The PRISMA (preferred reporting items for systematic reviews and meta- analyses) guidelines (35) were followed for reporting this systematic review. Ethics approval was not required. The protocol of this systematic review was registered on the SYRCLE website (https:// www.radboudumc.nl/Research/Organisationofresearch/Departments/ cdl/SYRCLE/Pages/Protocols).}

\footnotetext{
'Department of Neonatal Paediatrics, Princess Margaret Hospital for Children, Perth, Australia; ${ }^{2}$ Department of Neonatal Paediatrics, KEM Hospital for Women, Perth, Australia; ${ }^{3}$ Centre for Neonatal Research and Education, School of Paediatrics and Child Health, University of Western Australia, Perth, Australia. Correspondence: Gayatri Athalye-Jape (gayatri.jape@health.wa.gov.au)

Received 11 December 2016; accepted 2 September 2017; advance online publication 25 October 2017. doi:10.1038/pr.2017.218
} 
Search Strategy

The databases PubMed (http://www-ncbi-nlm-nih-gov, 1966August 2016), EMBASE (Excerpta Medica dataBASE) via Ovid (http://ovidsp.tx.ovid.com, 1980-August 2016), ISI Web of Science (v.5.21.1), http://webofscience.com, 1900-August 2016), and E-abstracts from the Pediatric Academic Society meetings (www. abstracts2view.com/pasall, 2000-August 2016), SCiELO (Scientific Electronic Library Online), and Lilacs (Literatura Latino Americana em Ciências da Saúde/Latin American database) databases were searched in December 2015 and August 2016. Abstracts of other conference proceedings such as Perinatal Society of Australia and New Zealand (PSANZ) and European Academy of Paediatric Societies (EAPS) were searched in EMBASE. The Animal Welfare Information Centre (http://awic.nal.usda.gov) data were searched in August 2016. Gray literature was searched using the national technical information services (http://www.ntis.gov/), Open Grey (http://www.opengrey.eu/), Grey net International (http://www.grey net.org/greysourceindex), and Trove (http://trove.nla.gov.au/). The reference lists of eligible studies and review articles were searched to identify additional studies. Reviewers GA-J and SR conducted the literature search independently. No language restrictions were applied.

\section{Search Terminology}

We searched PubMed using the following terms: ("necrotising enterocolitis" [All Fields] OR "enterocolitis, necrotizing"[MeSH] AND ("models, animal" [MeSH] AND "Probiotics" [Majr]. We also searched for ("Enterocolitis, Necrotizing"[Mesh]) AND "Models, Animal"[Majr], ((necrotizing enterocolitis) AND animal model AND probiotic, necrotizing enterocolitis and animal models, necrotizing enterocolitis and animal models and probiotics, experimental necrotizing enterocolitis and probiotics. The MeSH term "models, animal" was replaced with the following: "Rats"[Mesh], "Mice"[Mesh], "Swine"[Mesh], "Rabbits"[Mesh], and "Quail"[Mesh] for a further detailed search to enable inclusion of different species. Other databases were also searched using similar terms.

\section{Inclusion Criteria}

Only RCTs assessing effects of enteral probiotic supplementation (any dose, duration, frequency, type, and combination) vs. placebo/ control in validated animal models (rats, mice, piglets, rabbit, quail) of NEC (36) were eligible for inclusion. Studies assessing probiotic enriched formula, killed/inactivated probiotic, probiotic DNA, or probiotic conditioned medium vs. placebo/control/dam-fed animals were also included.

\section{Exclusion Criteria}

Studies assessing effects of probiotic supplemental in animal models that are not validated for NEC as it occurs in human preterm infants (e.g., colitis, or ischemia-reperfusion, in vitro studies) were excluded. Studies in hamsters, nematodes, and invertebrates were excluded as their relevance to NEC as it occurs in human preterm infants is uncertain (36). Narrative reviews, systematic reviews, case reports, letters, editorials, and commentaries were excluded but read to identify potential additional studies.

\section{Outcomes}

Primary outcomes included the incidence and/or severity of NEC. Secondary outcomes included the effects of probiotics or their derivatives on pathways involved in the pathogenesis of NEC, including (1) immunity, inflammation, and tissue injury, (2) intestinal barrier, (3) dysbiosis/gut microbiota, and (4) other mechanisms (e.g., epithelial growth factor, short-chain fatty acids, oligosaccharides, intestinal and liver fatty acid-binding protein, lysozyme and intestinal phospholipases, oxidative stress, plasma endotoxin, and organic acids).

\section{Data Extraction}

Authors GA-J and SR extracted the data independently by using a data collection form designed for this review. The number of animals in each group (probiotic, placebo, and control groups) with details of species, protocol for inducing NEC, and outcomes were entered into the form. All authors verified information about the study design and outcomes. Discrepancies during the data extraction process were resolved by discussion and consensus among all authors. We contacted authors for additional information and clarifications when details on incidence and severity of NEC were not available in the published manuscripts.

\section{Assessment of Risk of Bias in the Included Studies}

The risk of bias (ROB) was assessed using the SYRCLE "Risk of Bias" tool (37). This tool is based on the Cochrane ROB tool and incorporates aspects of bias that have a specific role in animal intervention studies. Authors GA-J and SR independently assessed the $\mathrm{ROB}$ in all domains, including random number generation, allocation concealment, random housing of animals, blinding of intervention and outcome assessors, selectivity of reporting, and other potential sources of bias. For each domain, the risk was assessed as low, high, or unclear based on the SYRCLE guidelines (37).

\section{Data Synthesis}

Meta-analysis was conducted in Review Manager 5.3 (Cochrane Collaboration, Nordic Cochrane Centre, Copenhagen, Denmark). A random-effects model (Mantel-Haenszel method) was used. Analysis was also conducted using the fixed-effects model to ensure that the results and conclusions were not influenced by the type of model used for the meta-analysis. Effect size was expressed as relative risk (RR) and 95\% confidence interval (CI). Statistical heterogeneity was assessed with $I^{2}$ statistic and by visual inspection of the forest plot (overlap of CIs). $I^{2}$ statistic values were interpreted according to the Cochrane Handbook guidelines as follows: $0-40 \%$ : might not be important; 30-60\%: may represent moderate heterogeneity; 50-90\%: may represent substantial heterogeneity; and 75-100\%: considerable heterogeneity (38). The risk of publication bias was assessed by visual inspection of the funnel plot (39).

\section{Subgroup Analysis}

Considering that the effects of an intervention can be animal species specific, a subgroup analysis was conducted for each of the animal species in the included studies.

\section{Sensitivity Analysis}

Analyses were conducted by including only studies where Bifidobacterium was present or absent, whether Lactobacillus was present or absent, studies in preterm animals, studies in term animals, and whether studies used single or multiple strain probiotics.

\section{Summary of Findings Table}

The key information about the quality of evidence, the magnitude of effect of the intervention, and the sum of available data on the main outcome are presented in the summary of findings table according to the Grades of Recommendation, Assessment, Development and Evaluation (GRADE) guidelines $(40,41)$.

\section{RESULTS}

The literature search retrieved 651 potentially relevant citations of which a total of 29 RCTs $(4,270)$ (Rodents: 23, Piglets: 3, Rabbits: 1, Quails: 2, $N \sim 2,310$ ) were considered eligible for inclusion. The flow diagram of study selection process is given in Supplementary Figure S1. Eleven studies were conducted in preterm animal models (42-52), 11 in term newborn animals (53-63), and the remaining 7 studied term 
animals at 2-4 weeks of age $(42,64-69)$. Twenty-five studies used single-strain probiotics $(42,43,46-48,50,52-70)$ and four used multi-strain probiotics $(44,45,49,51)$. The median sample size in the included studies was 72 (interquartile range: 36-96; range: 16-343). Twenty-one of the 29 trials reported the incidence of histologically proven NEC (42-51,53-59,64-67) (Supplementary Table S1). The remaining eight RCTs (52,60-63,68-70) assessed the pathways of benefits of probiotics in reducing NEC but did not give data on the incidence, severity, or histology of NEC (Supplementary Table S2). Khailova et al. (70) in 2010 and Khailova et al. (46) in 2009 were two separate publications from the same RCT with different primary outcomes. A total of 11 studies used Bifidobacteria $(43,46-48,50,53,54,57,62,65,68)$, 9 studies used Lactobacilli $(42,55,56,58,59,63,66,67,69), 3$ used both Bifidobacteria and Lactobacilli $(44,45,51), 3$ used Saccharomyces $(60,61,64), 1$ study used Lactococci $(52)$, and 1 study tested 5 different strains individually or in combination (49). The daily dose of probiotics ranged from 5 million to 24 billion colonyforming units (CFU). The heterogeneous nature of reporting of the dose (CFU/day, CFU/kg, and CFU/ml) made it difficult to estimate the median administered dose. The characteristics of the included studies (e.g., animal model details, probiotic protocol, outcomes) are given in Supplementary Tables S1 and S2.

\section{ROB Assessment Based on SYRCLE Guidelines}

Only one study reported on the method used for random sequence generation (54) Supplementary Table S3. Method used for allocation concealment was unclear in all studies. Only one study reported on the use of placebo (peptone water) (44). Castro et al. (59) mentioned that they used placebo; however, the placebo was milk. In 17/29 studies, pathologists who interpreted the intestinal histopathology for NEC were blinded. Only 7/29 studies reported that baseline characteristics were similar in the probiotic and no probiotic groups. None of the studies reported on measures that were used to house the animals randomly within the animal room. All studies reported complete follow-up data. The domain of selective reporting could not be assessed owing to lack of access to the original protocols of the included studies. Only two studies reported on sample size calculation $(49,51)$.

\section{Meta-Analysis}

Pooled data from 16/21 RCTs using random-effects model (Mantel-Haenszel) showed that probiotics significantly decreased the risk of NEC $(203 / 641$ (31.7\%) vs. $344 / 571$ (60.2\%); RR: 0.51; 95\% CI: 0.42, 0.62; $P<0.00001 ; I^{2}=44 \%$; number needed to treat: 4; 95\% CI: 2.9, 4.3) (Supplementary Figure S2). The incidence of severe NEC was reduced significantly in the probiotics vs. placebo group (72/331 (21.8\%) vs. $156 / 353$ (44.2\%); RR: $0.49,95 \%$ CI: $0.36,0.67$; $P<0.00001, I^{2}=20 \%$; number needed to treat: 5; 95\% CI: 3.4, 6.4) (Supplementary Figure S3). The results remained significant on analysis by fixed-effects model (1) NEC: RR:
0.51; 95\% CI: $0.44-0.59 ; P<0.00001$ and (2) Severe NEC: RR: 0.5, 95\% CI: $0.4,0.64, P<0.00001$.

\section{Publication Bias}

Visual inspection of the funnel plot indicated that publication bias was unlikely (Supplementary Figure S4).

\section{Results of Subgroup Analysis}

Subgroup analysis showed that probiotics were beneficial in all animal species, except piglets (Supplementary Figure S2).

\section{Results of Sensitivity Analysis}

Probiotic supplementation was beneficial in preterm as well as term gestation animals (Supplementary Table S4). The beneficial effects were noted whether Bifidobacterium was present or absent, Lactobacillus was present or absent, and in single-strain probiotic studies. Studies that used multiple strains showed benefit on fixed-effects model meta-analysis but not on random-effects model; however, the results for this analysis were heavily influenced by the only RCT that found probiotics to be harmful (45).

\section{Mechanisms of Benefits of Probiotics}

The mechanisms of benefits of probiotics observed in various studies are summarized in Supplementary Table S5. These include the following: modulation of the inflammatory response (Toll-like receptor 4 , nuclear factor- $\kappa \mathrm{B}$, reduction of plasma endotoxin levels), enhancement of the gut barrier (mucus production, synthesis of intercellular junction proteins, brush border enzyme activity), competitive inhibition of colonization by pathogens, secretion of antimicrobial peptides, production of short-chain fatty acid, reduction of oxidative stress, regulation of apoptosis and restitution, and modulating Paneth cell function.

\section{GRADE Evidence}

The positive aspects of the total evidence were the relatively large cumulative sample size, large effect size of benefit, narrow CIs around the effect size estimate, very low $P$-value for effect size estimate, absence of publication bias, mild statistical heterogeneity, and blinding of outcome assessors (Supplementary Table S6). The main limitation was the fact that majority of included studies had unclear ROB in many domains. Hence, overall the evidence was downgraded to moderate.

\section{DISCUSSION}

Our systematic review found that probiotic supplementation significantly reduced the incidence and severity of histologically proven NEC in animal models of the illness. The benefits of probiotics were consistent despite the heterogeneity of their strain, formulation, dose, and duration across four different species of term and preterm animals. These findings support the current thinking that, apart from their strain-specific effects, probiotics 'in general' provide a strain nonspecific protection toward NEC probably by sharing different 
pathways of benefits $(71,72)$. Sanders (73) suggested that "there may be a spectrum of probiotic functions which are strain specific but there are others, which are more general to larger groups of strains". Even though many meta-analyses of RCTs $(20-22,74)$ as well as non-RCTs (75-77) in human preterm infants have shown the benefits of probiotics, experts are still concerned about the routine use of probiotics in preterm very low birth weight (birth weight $<1,500 \mathrm{~g}$ ) infants. Some have pointed out that we still do not specifically know how probiotics work (78), some plead the need for much more basic science research in the field of probiotics in preterm infants (79), while others emphasize the need for further studies into the mechanism of actions of specific probiotic strains in models of immature intestine (80). Our systematic review is an attempt to address some of these concerns by synthesizing the evidence on the effects of probiotics in animal models of NEC. Such pathways are difficult to study in human infants, given that it is not feasible to obtain intestinal specimens from live human preterm infants. In addition, the meta-analysis results from animal studies are expected to add strength to the existing evidence and hopefully provide further reassurance to the clinicians.

The results of this meta-analysis are congruent with the multiple meta-analyses of RCTs and non-RCTs from human preterm infants that have found probiotics to reduce the incidence of NEC. These results are also congruent with the results of the ProPrems trial (81) that showed a statistically significant reduction in the incidence of NEC in the probiotic group (2.0\% vs. $4.4 \%$; RR $0.46,95 \%$ CI $0.23,0.93, P=0.03$; sample size 1,099). However, the other large RCT (PIPS trial) (82) did not show protection of NEC with probiotics (adjusted risk ratio 0.93 (95\% CI 0.68, 1.27; $N=1,315)$. The authors of the PIPS trial reported a very high crosscolonization rate $(20 \%$ at 2 weeks and $49 \%$ by 36 weeks postmenstrual age) in the placebo group in their trial. Such cross-colonization could have reduced the incidence of NEC even in the placebo group, thereby leading to no statistically significant differences between the two groups. Hence the authors of the PIPS trial suggested that any future RCTs should consider cluster RCT design to avoid the problem of cross-contamination (82).

Although the evidence on the beneficial effects of probiotics in preterm infants is mounting, further research is necessary to identify the optimal strain/s, optimal dose, and duration of supplementation. The RCTs addressing these questions do not require the use of placebo; they can be answered with head-to-head comparisons (e.g.,: single strain vs. multiple strain; high dose vs. low dose; short duration vs. long duration of supplementation, bifidobacteria vs. lactobacillus, one type of bifidobacteria vs. other, and many more).

The strengths and limitations of our review need to be discussed. To our knowledge, this is the first systematic review and meta-analysis of studies assessing the effects of probiotics in animal models of NEC. Our meta-analysis of data from animal models adds further strength to the evidence from studies in human preterm infants. The detailed synthesis of evidence regarding the various mechanisms of benefits of probiotics is an additional strength of this review. This is also the first such comprehensive review in neonatal medicine. The validity of its results is high considering the methodology based on the SYRCLE protocol (WWW.SYRCLE.NL), comprehensive literature search, and the use of PRISMA guidelines (35) for reporting. The significance of our results cannot be overemphasized considering the large effect size, extremely low $P$-values that almost rule out the role of chance alone, low risk for publication bias, minimal-to-moderate statistical heterogeneity, and their consistency on analysis by both random-effects model and fixed-effects model.

The limitations of our review include smaller sample size of individual studies, lack of power calculations in majority of the studies, and the fact that most studies carried unclear ROB on various domains. In the context of sample sizes, we do accept the limitations in carrying out adequately powered animal studies considering the costs, logistics, and ethical issues. Our view on this issue is shared by other investigators $(83,84)$. Another limitation of our review was the inability to fully evaluate the safety of probiotics in animal models. However, it is reassuring to note that $>40$ RCTs $(n \sim 11,000)$ in human preterm infants have not shown increased risk of late-onset sepsis and mortality. If at all, probiotics have significantly reduced the risk of these outcomes $(19-22,80,85)$. The recently published largest RCT (PIPS trial) did not find Bifidobacterium species cultured from any normally sterile site (81). Although these findings are reassuring, one should not be complacent considering the case reports of probiotic sepsis $(86,87)$. Rigorous quality control of the probiotic product and routine monitoring for probiotic sepsis are therefore critical (88).

Extrapolating results from animal models to human preterm infants may not be appropriate as the effects of an intervention could be species specific $(82-84,89)$. For example, intraperitoneal pentoxifylline prevented NEC in a rat model but not in a rabbit model $(90,91)$. Animal models of NEC may not reflect the multifactorial (e.g., intrauterine growth restriction, sepsis, patent ductus arteriosus, indomethacin, no enteral intake, prolonged parenteral nutrition) pathogenesis of classical late-onset NEC in very/extremely preterm infants. The developmental anatomy and physiology of the gastrointestinal tract of piglets is comparable to that of the human preterm infants, but for inducing NEC, the model often includes only formula feeding to which the animals are very sensitive $(44,45)$. The rat pup model utilizes a combination of insults, including hypoxia, hypothermia, and formula feeds $(43,46-49,55,57,58)$, which is not what commonly leads to NEC in human preterm infants (92). However, it is reassuring to know that our findings are similar to the results of systematic reviews of probiotic RCTs and non-RCTs (before vs. after routine use) in human preterm infants $(20-22,74-76)$.

Two of the included studies in our review used probioticconditioned medium and found benefits in decreasing NEC $(51,62)$. The benefits of probiotic conditioned medium are 
important as this strategy may help in avoiding the risk of probiotic sepsis, antibiotic resistance, and the need for cold chain. Further studies are essential for assessing its advantages and limitations. For example, probiotic conditioned medium may not be as effective as live/killed-inactivated strains, effects of probiotic conditioned medium from different strains and/ or combination may differ, and there may be logistical issues related to mass production.

In summary, our systematic review of animal studies provides robust evidence supporting the benefits of probiotics in reducing the risk of NEC in preterm infants. Despite their limitations, the importance of studies in animal models in guiding research and clinical practice in the field of probiotics and NEC is emphasized. Addressing the need for raising the gold standard by improving the design, conduct, and reporting of animal studies is important (93-96). We believe that our results are a significant contribution toward advancing knowledge in the field of probiotic supplementation for reducing the risk of NEC in preterm infants.

\section{SUPPLEMENTARY MATERIAL}

Supplementary Information accompanies this paper at http://www.nature. $\mathrm{com} / \mathrm{pr}$

Disclosure: The authors declare no conflict of interest.

\section{REFERENCES}

1. Neu J, Walker WA. Necrotizing enterocolitis. N Engl J Med 2011;364: 255-64.

2. Lin PW, Stoll BJ. Necrotising enterocolitis. Lancet 2006;368:1271-83.

3. Griffin IJ, Tancredi DJ, Bertino E, et al. Postnatal growth failure in very low birthweight infants born between 2005 and 2012. Arch Dis Child Fetal Neonatal Ed 2016;101:F50-5.

4. Thyoka M, De Coppi P, Eaton S, et al. Advanced necrotizing enterocolitis. Part 1: Mortality. Eur J Pediatr Surg 2012;22:8-12.

5. Kelleher J, Mallick H, Soltau TD, et al. Mortality and intestinal failure in surgical necrotizing enterocolitis. J Pediatr Surg 2013;48:568-72.

6. Rees CM, Pierro A, Eaton S. Neurodevelopmental outcomes of neonates with medically and surgically treated necrotizing enterocolitis. Arch Dis Child Fetal Neonatal Ed 2007;92:F193-8.

7. Shah TA, Meinzen-Derr J, Gratton T, et al. Hospital and neurodevelopmental outcomes of extremely low-birth-weight infants with necrotizing enterocolitis and spontaneous intestinal perforation. J Perinatol 2012;32: $552-8$.

8. Wadhawan R, Oh W, Hintz SR, et al. NICHD Neonatal Research Network. Neurodevelopmental outcomes of extremely low birth weight infants with spontaneous intestinal perforation or surgical necrotizing enterocolitis. J Perinatol 2014;34:64-70.

9. Pike K, Brocklehurst P, Jones D, et al. Outcomes at 7 years for babies who developed neonatal necrotising enterocolitis: the ORACLE Children Study. Arch Dis Child Fetal Neonatal Ed 2012;97:F318-22.

10. Schulzke SM, Deshpande GC, Patole SK. Neurodevelopmental outcomes of very low-birth-weight infants with necrotizing enterocolitis: a systematic review of observational studies. Arch Pediatr Adolesc Med 2007;161:583-90.

11. Hintz SR, Kendrick DE, Stoll BJ, et al. Neurodevelopmental and growth outcomes of extremely low birth weight infants after necrotizing enterocolitis. Pediatrics 2005;115:696e703.

12. Fitzgibbons SC, Ching Y, Yu D, et al. Mortality of necrotizing enterocolitis expressed by birth weight categories. J Pediatr Surg. 2009;44: $1072-5$.
13. Luig M, Lui KNSW \& ACT NICUS Group. Epidemiology of necrotizing enterocolitis-Part I: Changing regional trends in extremely preterm infants over 14 years. J Paediatr Child Health 2005;41:169-73.

14. Salhab WA, Perlman JM, Silver L, et al. Necrotizing enterocolitis and neurodevelopmental outcome in extremely low birth weight infants $<1000$ g. J Perinatol 2004;24:534-40.

15. Bisquera JA, Cooper TR, Berseth CL. Impact of necrotizing enterocolitis on length of stay and hospital charges in very low birth weight infants. Pediatrics 2002;109:423-8.

16. Ganapathy V, Hay JW, Kim JH, et al. Long term healthcare costs of infants who survived neonatal necrotizing enterocolitis: a retrospective longitudinal study among infants enrolled in Texas Medicaid. BMC Pediatr 2013;13:127.

17. Torrazza RM, Li N, Neu J. Decoding the enigma of necrotizing enterocolitis in premature infants. Pathophysiology 2014;21:21-7.

18. Choi YY. Necrotizing enterocolitis in newborns: update in pathophysiology and newly emerging therapeutic strategies. Korean J Pediatr 2014;57:505-13.

19. AlFaleh K, Anabrees J. Probiotics for prevention of necrotizing enterocolitis in preterm infants. Cochrane Database Syst Rev 2014;4:CD005496.

20. Lau CS, Chamberlain RS. Probiotic administration can prevent necrotizing enterocolitis in preterm infants: a meta-analysis. J Pediatr Surg 2015;50:1405-2.

21. Sawh SC, Deshpande S, Jansen S, et al. Prevention of necrotizing enterocolitis with probiotics: a systematic review and meta-analysis. PeerJ 2016;4:e2429.

22. Wang XL, Li X, Kang L, et al. Prophylactic probiotics for preventing necrotizing enterocolitis and reducing mortality in very low birth weight infants: a meta-analysis. Zhongguo Dang Dai Er Ke Za Zhi 2015;17:852-.

23. Rao RK, Samak G. Protection and restitution of gut barrier by probiotics: nutritional and clinical implications. Curr Nutr Food Sci 2013;9:99-107.

24. Ewaschuk JB, Diaz H, Meddings L, et al. Secreted bioactive factors from Bifidobacterium infantis enhance epithelial cell barrier function. Am J Physiol Gastrointest Liver Physiol 2008;295:G1025-34.

25. Walker A. Intestinal colonization and programming of the intestinal immune response. J Clin Gastroenterol 2014;48:S8-11.

26. Garrido D, Barile D, Mills DA. A molecular basis for bifidobacterial enrichment in the infant gastrointestinal tract. Adv Nutr 2012;3: 415S-21S.

27. Di Mauro A, Neu J, Riezzo G, et al. Gastrointestinal function development and microbiota. Ital J Pediatr 2013;39:15.

28. Denayer T, Stöhr T, Van Roy M. Animal models in translational medicine: validation and prediction. New Horiz Transl Med 2014;2:5-11.

29. Ritskes-Hoitinga M, Leenaars M, Avey M, et al. Systematic reviews of preclinical animal studies can make significant contributions to health care and more transparent translational medicine [editorial]. Cochrane Database Syst Rev 2014;3:ED000078.

30. Pound P, Ebrahim S, Sandercock P, et al. Where is the evidence that animal research benefits humans? BMJ 2004;328:514-7.

31. Gopalakrishnan S, Ganeshkumar P. Systematic reviews and metaanalysis: understanding the best evidence in primary healthcare. J Family Med Prim Care 2013;2:9-14.

32. Nordmann AJ, Kasenda B, Briel M. Meta-analyses: what they can and cannot do. Swiss Med Wkly 2012;142:w13518.

33. Hooijmans CR, Ritskes-Hoitinga M. Progress in using systematic reviews of animal studies to improve translational research. PLoS Med 2013;10: e1001482.

34. Sandercock P, Roberts I. Systematic reviews of animal experiments. Lancet 2002;360:586.

35. Moher D, Liberati A, Tetzlaff J, et al. Preferred reporting items for systematic reviews and meta-analyses: the PRISMA statement. Ann Intern Med 2009;151:264-9.

36. Lu P, Sodhi CP, Jia H, et al. Animal models of gastrointestinal and liver diseases. Animal models of necrotizing enterocolitis: pathophysiology, translational relevance, and challenges. Am J Physiol Gastrointest Liver Physiol 2014;306:G917-28. 
37. Hooijmans CR, Rovers MM, de Vries RB, et al. SYRCLE's risk of bias tool for animal studies. BMC Med Res Methodol 2014;14:43.

38. Higgins JPTG, Higgins S Cochrane handbook of systematic reviews of interventions. Version 5.1.0. 2011; The Cochrane Collaboration available at www.handbook.cochrane.org Accessed 16 August 2016.

39. Sterne JA, Egger M. Funnel plots for detecting bias in meta-analysis: guidelines on choice of axis. J Clin Epidemiol 2001;54:1046-55.

40. Guyatt GH, Oxman AD, Santesso N, et al. GRADE guidelines: 12. Preparing summary of findings tables binary outcomes. J Clin Epidemiol 2013;66:158-72.

41. Wei D, Tang K, Wang Q, et al. The use of GRADE approach in systematic reviews of animal studies. J Evid Based Med 2016;9:98-104.

42. Good M, Sodhi CP, Ozolek JA, et al. Lactobacillus rhamnosus HN001 decreases the severity of necrotizing enterocolitis in neonatal mice and preterm piglets: evidence in mice for a role of TLR9. Am J Physiol Gastrointest Liver Physiol 2014;306:G1021-32.

43. Satoh $\mathrm{T}$, Izumi $\mathrm{H}$, Iwabuchi $\mathrm{N}$, et al. Bifidobacterium breve prevents necrotising enterocolitis by suppressing inflammatory responses in a preterm rat model. Benef Microbes 2015;7:1-8.

44. Siggers RH, Siggers J, Boye M, et al. Early administration of probiotics alters bacterial colonization and limits diet-induced gut dysfunction and severity of necrotizing enterocolitis in preterm pigs. J Nutr 2008;138:1437-44.

45. Cilieborg MS, Thymann T, Siggers R, et al. The incidence of necrotizing enterocolitis is increased following probiotic administration to preterm pigs. J Nutr 2011;141:223-30.

46. Khailova L, Dvorak K, Arganbright KM, et al. Bifidobacterium bifidum improves intestinal integrity in a rat model of necrotizing enterocolitis. Am J Physiol Gastrointest Liver Physiol 2009;297:G940-9.

47. Underwood MA, Arriola J, Gerber CW, et al. Bifidobacterium longum subsp. infantis in experimental necrotizing enterocolitis: alterations in inflammation, innate immune response, and the microbiota. Pediatr Res 2014;76:326-3.

48. Underwood MA, Kananurak A, Coursodon CF, et al. Bifidobacterium bifidum in a rat model of necrotizing enterocolitis: antimicrobial peptide and protein responses. Pediatr Res 2012;71:546-1.

49. Wu SF, Chiu HY, Chen AC, et al. Efficacy of different probiotic combinations on death and necrotizing enterocolitis in a premature rat model. J Pediatr Gastroenterol Nutr 2013;57:23-8.

50. Ling X, Linglong P, Weixia D, et al. Protective effects of bifidobacterium on intestinal barrier function in LPS-induced enterocyte barrier injury of Caco-2 monolayers and in a rat NEC model. PLoS ONE 2016;11: e0161635.

51. Shiou SR, Yu Y, Guo Y, et al. Synergistic protection of combined probiotic conditioned media against neonatal necrotizing enterocolitis-like intestinal injury. PLoS ONE 2013;8:e65108.

52. McVay MR, Boneti C, Habib CM, et al. Formula fortified with live probiotic culture reduces pulmonary and gastrointestinal bacterial colonization and translocation in a newborn animal model. J Pediatric Surg 2008;43:25-9.

53. Bergmann KR, Liu SX, Tian R, et al. Bifidobacteria stabilize claudins at tight junctions and prevent intestinal barrier dysfunction in mouse necrotizing enterocolitis. Am J Pathol 2013;182:1595-606.

54. Zhou W, Lv H, Li MX, et al. Protective effects of bifidobacteria on intestines in newborn rats with necrotizing enterocolitis and its regulation on TLR2 and TLR4. Genet Mol Res 2015;14:11505-4.

55. Liu Y, Fatheree NY, Mangalat N, et al. Lactobacillus reuteri strains reduce incidence and severity of experimental necrotizing enterocolitis via modulation of TLR4 and NF-kappaB signaling in the intestine. Am J Physiol Gastrointest Liver Physiol 2012;302:G608-17.

56. Goncalves FL, Soares LM, Figueira RL, et al. Evaluation of the expression of I-FABP and L-FABP in a necrotizing enterocolitis model after the use of Lactobacillus acidophilus. J Pediatr Surg 2015;50:543-9.

57. Caplan MS, Miller-Catchpole R, Kaup S, et al. Bifidobacterial supplementation reduces the incidence of necrotizing enterocolitis in a neonatal rat model. Gastroenterology 1999;117:577-83.
58. Olson JK, Rager TM, Navarro JB, et al. Harvesting the benefits of biofilms: a novel probiotic delivery system for the prevention of necrotizing enterocolitis. J Pediatr Surg 2016;51:936-41.

59. Castro E, Cofré J, Mellado JP, et al. Induction of necrotizing enterocolitis in non-premature Sprague-Dawley rats and the effect of administering breast milk-isolated Lactobacillus salivarius LPLM-O1. Food Nutr Sci 2014;5:1255-60.

60. D'Souza A, Cai CL, Kumar D, et al. Cytokines and Toll-like receptor signaling pathways in the terminal ileum of hypoxic/hyperoxic neonatal rats: benefits of probiotics supplementation. Am J Transl Res 2012;4: 187-97.

61. D'Souza A, Fordjour L, Ahmad A, et al. Effects of probiotics, prebiotics, and synbiotics on messenger RNA expression of caveolin-1, NOS, and genes regulating oxidative stress in the terminal ileum of formula-fed neonatal rats. Pediatr Res 2010;67:526-31.

62. Weng M, Ganguli K, Zhu W, et al. Conditioned medium from Bifidobacteria infantis protects against Cronobacter sakazakii-induced intestinal inflammation in newborn mice. Am J Physiol Gastrointest Liver Physiol 2014;306:G779-87.

63. Hunter CJ, Williams M, Petrosyan M, et al. Lactobacillus bulgaricus prevents intestinal epithelial cell injury caused by Enterobacter sakazakiiinduced nitric oxide both in vitro and in the newborn rat model of necrotizing enterocolitis. Infect Immun 2009;77:1031-43.

64. Akisu M, Baka M, Yalaz M, et al. Supplementation with Saccharomyces boulardii ameliorates hypoxia/reoxygenation-induced necrotizing enterocolitis in young mice. Eur J Pediatr Surg 2003;13:319-23.

65. Butel MJ, Roland N, Hibert A, et al. Clostridial pathogenicity in experimental necrotising enterocolitis in gnotobiotic quails and protective role of bifidobacteria. J Med Microbiol 1998;47:391-9.

66. Liu Y, Tran DQ, Fatheree NY, et al. Lactobacillus reuteri DSM 17938 differentially modulates effector memory $\mathrm{T}$ cells and Foxp3+ regulatory $\mathrm{T}$ cells in a mouse model of necrotizing enterocolitis. Am J Physiol Gastrointest Liver Physiol 2014;307:G177-86.

67. Mirpuri J, Sotnikov I, Myers L, et al. Lactobacillus rhamnosus (LGG) regulates IL-10 signaling in the developing murine colon through upregulation of the IL-10R2 receptor subunit. PLoS ONE 2012;7: e51955.

68. Catala I, Butel MJ, Bensaada M, et al. Oligofructose contributes to the protective role of bifidobacteria in experimental necrotising enterocolitis in quails. J Med Microbiol 1999;48:89-94.

69. Lin PW, Myers LE, Ray L, et al. Lactobacillus rhamnosus blocks inflammatory signaling in vivo via reactive oxygen species generation. Free Radic Biol Med 2009;47:1205-1.

70. Khailova L, Mount Patrick SK, Arganbright KM, et al. Bifidobacterium bifidum reduces apoptosis in the intestinal epithelium in necrotizing enterocolitis. Am J Physiol Gastrointest Liver Physiol 2010;299: G1118-27.

71. Ganguli K, Walker WA. Probiotics in the prevention of necrotizing enterocolitis. J Clin Gastroenterol 2011;45 (Suppl): S133-8.

72. Vandenplas Y, Veereman-Wauters G. Probiotics: fishing in the ocean. J Pediatr Gastroenterol Nutr 2012;54:4-5.

73. Sanders ME Is it time to consider generic probiotic effects? California Dairy Research Foundation 1/3/2011. http://cdrf.org/2013/03/01/is-ittime-to-consider-generic-probiotic-effects/. Accessed 9 October 2016.

74. Deshpande G, Rao S, Patole S, et al. Updated meta-analysis of probiotics for preventing necrotizing enterocolitis in preterm neonates. Pediatrics 2010;125:921-30.

75. Olsen R, Greisen G, Schrøder M, et al. Prophylactic probiotics for preterm infants: a systematic review and meta-analysis of observational studies. Neonatology 2016;109:105-2.

76. Dermyshi E, Wang Y, Yan C, et al. The "Golden Age" of probiotics: a systematic review and meta-analysis of randomized and observational studies in preterm infants. Neonatology 2017;112:9-23.

77. Neu J. Routine probiotics for premature infants: let's be careful!. J Pediatr 2011;158:672-4. 
78. Modi N. Probiotics and necrotising enterocolitis: the devil (as always) is in the detail. Neonatology 2014;105:71-3.

79. Neu J. Probiotics and necrotizing enterocolitis. Clin Perinatol 2014;41: 967-78.

80. Jacobs SE, Tobin JM, Opie GF, et al. Probiotic effects on late-onset sepsis in very preterm infants: a randomized controlled trial. Pediatrics 2013;132:1055-62.

81. Costeloe K, Hardy P, Juszczak E, et al. Bifidobacterium breve BBG-001 in very preterm infants: a randomised controlled phase 3 trial. Lancet 2016;387:649-0.

82. Sangild PT, Ney DM, Sigalet DL, et al. Animal models of gastrointestinal and liver diseases. Animal models of infant short bowel syndrome: translational relevance and challenges. Am J Physiol Gastrointest Liver Physiol 2014;307:G1147-68.

83. Moran CJ, Ramesh A, Brama PA, et al. The benefits and limitations of animal models for translational research in cartilage repair. J Exp Orthop 2016;3:1.

84. Martinez MInterspecies differences in physiology and pharmacology: extrapolating preclinical data to human populationsIn:Rogge MC, Taft DReds. Preclinical Drug Development 2. Boca Raton, FL, USA: Taylor and Francis Group, 2009: 35-70.

85. Rao SC, Athalye-Jape GK, Deshpande GC, et al. Probiotic supplementation and late-onset sepsis in preterm infants: a meta-analysis. Pediatrics 2016;137:e20153684.

86. Zbinden A, Zbinden R, Berger C, et al. Case series of Bifidobacterium longum bacteremia in three preterm infants on probiotic therapy. Neonatology 2015;107:56-9.

87. Bertelli C, Pillonel T, Torregrossa A, et al. Bifidobacterium longum bacteremia in preterm infants receiving probiotics. Clin Infect Dis 2015;60:924-7.
88. Centers for Disease Control and Prevention Fatal gastrointestinal mucormycosis in an infant following use of contaminated $\mathrm{ABC}$ dophilus powder from Solgar Inc, 2014. Accessed 3 August 2017. Available from http://www.cdc.gov.pklibresources.health.wa.gov.au/ fungal/rhizopus-investigation.html.

89. Barrington KJ, Ryan CA, Finer NN. Effects of magnesium sulfate in a newborn piglet meconium aspiration model. J Perinatol 2000;20: 373-8.

90. Travadi J, Patole S, Charles A, et al. Pentoxifylline reduces the incidence and severity of necrotizing enterocolitis in a neonatal rat model. Pediatr Res 2006;60:185-9.

91. Erdener D, Bakirtas F, Alkanat M, et al. Pentoxifylline does not prevent hypoxia/reoxygenation-induced necrotizing enterocolitis. An experimental study. Biol Neonate 2004;86:29-33.

92. Neu J. Necrotizing enterocolitis: the mystery goes on. Neonatology 2014;106:289-95.

93. Baker D, Lidster K, Sottomayor A, et al. Two years later: journals are not yet enforcing the ARRIVE guidelines on reporting standards for preclinical animal studies. PLoS Biol 2014;12:e1001756.

94. Avey MT, Fenwick N, Griffin G. The use of systematic reviews and reporting guidelines to advance the implementation of the 3Rs. J Am Assoc Lab Anim Sci 2015;54:153-62.

95. Burdge GC. Improving standards for reporting studies involving humans and experimental animals in the British Journal of Nutrition and in the Journal of Nutritional Science. Br J Nutr 2014;112:1423-4.

96. Hooijmans CR, de Vries R, Leenaars M, et al. Improving planning, design, reporting and scientific quality of animal experiments by using the Gold Standard Publication Checklist, in addition to the ARRIVE guidelines. $\mathrm{Br}$ J Pharmacol 2011;162:1259-60. 